\title{
Rapid On-Site Evaluation by Endosonographers during Endoscopic Ultrasonography-Guided Fine-Needle Aspiration for Diagnosis of Gastrointestinal Stromal Tumors
}

\author{
Takashi Tamura', Yasunobu Yamashita', Kazuki Ueda', Yuki Kawaji', Masahiro Itonaga', Shin-ichi Murata², Kaori Yamamoto², \\ Takeichi Yoshida ${ }^{1}$, Hiroki Maeda', Takao Maekita', Mikitaka Iguchi' ${ }^{1}$, Hideyuki Tamai', Masao Ichinose ${ }^{1}$ and Jun Kato ${ }^{1}$ \\ ${ }^{1}$ Second Department of Internal Medicine, ${ }^{2}$ Department of Human Pathology, Wakayama Medical University, Wakayama, Japan
}

Background/Aims: Endoscopic ultrasonography-guided fine-needle aspiration (EUS-FNA) has been used to diagnose gastrointestinal submucosal tumors (SMTs). Although rapid on-site evaluation (ROSE) has been reported to improve the diagnostic accuracy of EUS-FNA for pancreatic lesions, on-site cytopathologists are not routinely available. Given this background, the usefulness of ROSE by endosonographers themselves for pancreatic tumors has also been reported. However, ROSE by endosonographers for diagnosis of SMT has not been reported. The aim of this study was to evaluate the diagnostic accuracy of EUS-FNA with ROSE by endosonographers for SMT, focusing on diagnosis of gastrointestinal stromal tumor (GIST), compared with that of EUS-FNA alone. Methods: Twenty-two consecutive patients who underwent EUS-FNA with ROSE by endosonographers for SMT followed by surgical resection were identified. Ten historical control subjects who underwent EUS-FNA without ROSE were used for comparison. Results: The overall diagnostic accuracy for SMT was significantly higher in cases with than without ROSE (100\% vs. 80\%, $p=0.03$ ). The number of needle passes by FNA with ROSE by endosonographers tended to be fewer, although accuracy was increased ( $3.3 \pm 1.3$ vs. $5.9 \pm 3.8, p=0.06)$.

Conclusions: ROSE by endosonographers during EUS-FNA for SMT is useful for definitive diagnosis, particularly for GIST.

Clin Endosc 2017;50:372-378

Key Words: Endoscopic ultrasound-guided fine needle aspiration; Gastrointestinal stromal tumor; Endosonographer; On-site cytology

\section{INTRODUCTION}

The cytomorphological distinction of gastrointestinal stromal tumors (GISTs) from their benign counterparts, such as leiomyomas and schwannomas, is sometimes difficult, because those tumors are commonly comprised of spindle-shaped cells. Therefore, diagnosing GIST with only cytology smears

Received: June 22, 2016 Revised: October 5, 2016

Accepted: October 10, 2016

Correspondence: Yasunobu Yamashita

Second Department of Internal Medicine, Wakayama Medical University, 811-1 Kimiidera, Wakayama 641-8509, Japan

Tel: +81-73-447-2300, Fax: +81-73-445-3616, E-mail: yasunobu@wakayama-med.ac.jp

(c) This is an Open Access article distributed under the terms of the Creative Commons Attribution Non-Commercial License (http://creativecommons.org/ licenses/by-nc/3.0) which permits unrestricted non-commercial use, distribution, and reproduction in any medium, provided the original work is properly cited. may be inaccurate, and immunohistochemical staining is required. In particular, recent application of immunohistochemical staining of a c-kit proto-oncogene product that is overexpressed in nearly all GISTs could efficiently distinguish these neoplasms from leiomyomas and schwannomas. ${ }^{1} \mathrm{Al}-$ though sufficient materials are required for immunostaining, it is sometimes difficult to know whether sufficient materials are obtained during the endoscopic ultrasonography-guided fine-needle aspiration (EUS-FNA) procedure.

Recently, rapid on-site evaluation (ROSE) has been reported to improve the diagnostic accuracy rate of EUS-FNA for pancreatic solid masses. ROSE is a service to check for a sufficient amount of cells harvested through EUS-FNA by on-site pathologists and cytotechnologists. It has been reported that ROSE raises the specimen collection rate of EUS-FNA against pancreatic solid masses $15 \%$ and improves the diagnostic ac- 
curacy for pancreatic solid masses. ${ }^{2}$ However, it is a challenge to always have cytopathologists available for EUS-FNA in most institutions because of the time and cost required for ROSE. This problem is resolved through endosonographers performing ROSE on their own. Although the usefulness of ROSE by endosonographers has been reported in EUS-FNA of pancreatic masses, ${ }^{3,4}$ ROSE by endosonographers for EUSFNA of gastrointestinal submucosal tumors (SMTs) has not
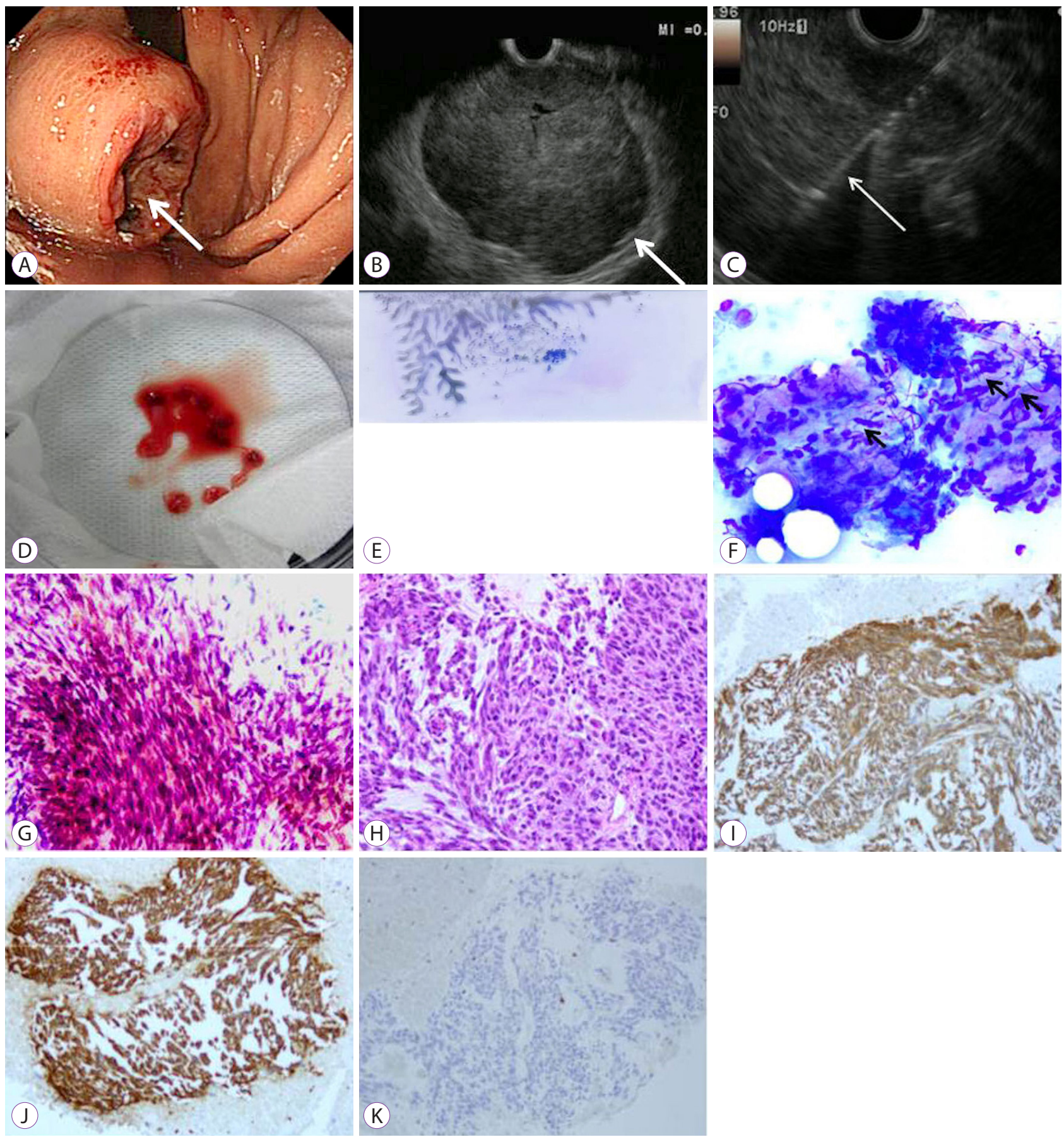

Fig. 1. Specimen processing for endoscopic ultrasonography-guided fine-needle aspiration (EUS-FNA) with rapid on-site evaluation (ROSE) by endosonographers. (A) A submucosal tumor (SMT) of the stomach is seen as a protuberant lesion (arrow) with an ulcer by upper endoscopy. (B) B-mode EUS images reveal a low echoic lesion (arrow). (C) The aspiration needle (arrow) punctures the mass. (D) The specimens are transferred to a watch glass. (E) The cytological slides processed from whitish specimens per puncture. (F) May-Giemsa staining using the Diff-Quik method detects spindle-shaped cells (arrows) $(\times 400)$. (G) Papanicolaou staining for cytological smear shows spindle-shaped cells $(\times 400)$. (H) Hematoxylin and eosin staining for histological examination shows spindle-shaped cells $(\times 200)$. (I, J, K) Immunohistochemical staining with c-kit (I), CD34 (J), and S-100 (K). Positive staining is observed with c-kit and CD34, whereas S-100 staining is negative $(\times 200)$. 
yet been evaluated.

The aim of this study was to identify the diagnostic accuracy of EUS-FNA with ROSE by endosonographers for gastrointestinal SMT, particularly focusing on GIST, compared with only EUS-FNA.

\section{MATERIALS AND METHODS}

\section{Patients}

The first case of EUS-FNA for gastrointestinal SMT at our institute (Wakayama Medical University Hospital, Wakayama, Japan) was performed in January 2009, and the procedure was modified with ROSE in February 2013. All procedures performed without ROSE before February 2013 were defined as period 1, while procedures accompanied by ROSE from February 2013 were defined as period 2 . The data of consecutive patients who underwent EUS-FNA with subsequent surgery during periods 1 and 2 were collected. Clinical characteristics of the patients and tumors and details of the EUS-FNA procedures were obtained from the medical charts. The final pathological diagnoses of surgically resected specimens were also evaluated.

The protocol of this study was approved by the ethics committee of Wakayama Medical University. As this was a retrospective study without any intervention, written and informed consent from each patient was not required.

\section{EUS-FNA}

EUS-FNA was performed on patients while their pulse was monitored after being sedated with diazepam and pentazocine. EUS-FNA was performed using convex-type endoscopes (GF-UCT260, GF-UCT240; Olympus, Tokyo, Japan) by three endosonographers, all of whom had at least 10 years of EUS experience and sufficient experience in more than 100 pancreatic EUS-FNA procedures before this study. We used Doppler imaging to avoid vascular structures for the puncture during the procedure. Puncture and aspiration were performed using standard 19- or 22-gauge (G) needles (Expect; Boston Scientific Japan, Tokyo, Japan). The 19-G needle was used at the beginning of the procedure in period 1. In the event of technical difficulties with the $19-\mathrm{G}$ needle, a $22-\mathrm{G}$ needle was used. The 19-G needle was also used for the first two cases in period 2. As the 22-G needle was deemed more suitable for the procedure based on the results of the initial two cases in period 2, it was used for subsequent cases in period 2. After puncture, the inner cylinder of the FNA needle was pulled out. While applying a syringe with a suction pressure of $20 \mathrm{~mL}$ to the FNA needle, the inside of the mass was repeatedly penetrated between 10 and 20 times to collect the cells (Fig. 1A-C). After the needle was withdrawn from the endoscope channel, the stylet was reintroduced, and the specimen was transferred to a watch glass.
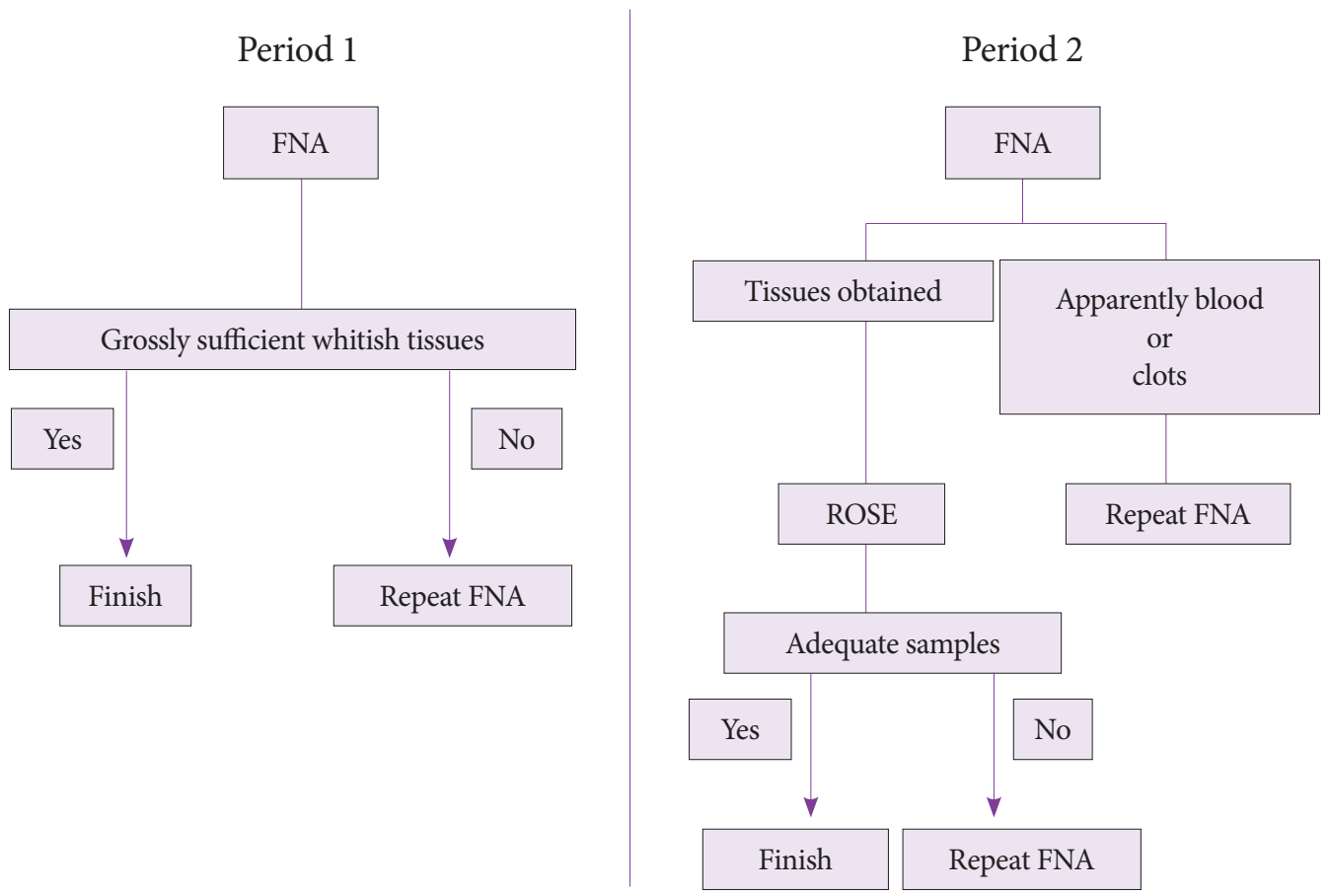

Fig. 2. Sequence of endoscopic ultrasonography-guided fine-needle aspiration (EUS-FNA) procedure with and without rapid on-site evaluation (ROSE). 


\section{Specimen processing for FNA}

As on-site cytotechnologists or cytopathologists were not available for ROSE at our institute, two endosonographers who had undergone extensive training in the processing techniques and reviewed cytological smears alongside a pathologist prepared smears using the conventional direct smear method prior to period 2. Specifically, two endosonographers were educated for 1 month on the cytological findings of normal gut wall (contamination), nonpathologic and pathologic disease entities, and adequate specimens for ROSE by a pathologist. Finally, a post-training test for defining adequate specimens was administered using an EUS-FNA sample of SMT in period 1 by the pathologist. The sequences of the procedures for periods 1 and 2 are depicted in Fig. 2.

In period 1 , the procedure involved repeated punctures with a needle pass until whitish specimens were obtained, characterized by the gross appearance of tissues free of blood and clots. The whitish specimens were placed in $10 \%$ formalin for fixation in the endoscopy suite.

In period 2, cytological slides were processed from the puncture specimens, except for cases appearing to have only blood or clots in the endoscopy suite. Some of the specimens were smeared using two slides. In order to avoid tissue destruction caused by drying, one of the slides with a smear was immediately fixed with alcohol, while the other slide was air dried for 1 minute. The slides then underwent May-Giemsa staining using the Diff-Quik method (Sysmex Inc., Kobe, Japan) and immediately evaluated by an endosonographer to determine whether sufficient specimens were collected using a microscope in the endoscopy suite (Fig. 1D-F). When the endosonographer indicated that a sufficient number of spindle-shaped cells were present, the procedure was stopped. If the sample did not contain spindle-shaped cells, the procedure was stopped when sufficient cellular contents that were not normal gut wall (contamination) and inflammatory cells appeared twice. The remaining specimens were fixed in $10 \%$ formalin for pathological examination. If the aspirate was found to contain only inflammatory cells, EUS-FNA was repeated in a different area of the puncture until adequate samples were obtained.

\section{Pathological diagnoses}

All diagnoses were made by one pathologist and one cytologist without knowledge of the EUS-FNA procedure in detail. Alcohol-fixed smears were stained using Papanicolaou stain in the Pathology Department (Fig. 1G). Both the EUS-FNA and surgical resection specimens were fixed in 10\% formalin, and tissue blocks were embedded in paraffin. Histopathological analyses were performed by staining tissue sections with hematoxylin and eosin (Fig. 1H). Immunohistochemical staining was subsequently performed on the representative histological sections of the tumor using commercially available antibodies against c-kit (CD117), CD34, S-100, and smooth muscle actin (SMA) (Fig. 1I-K). Diagnosis of GIST was made when pathologic examination showed spindle-shaped or epithelioid cells that stained positively for c-kit.

\section{Statistical analyses}

The $\chi^{2}$ test or Fisher's exact test and the McNemar's test were used to compare categorical variables. The Mann-Whitney $U$ test or Student's $t$-test was used for continuous variables. The difference was considered significant when the $p$-value was less than 0.05. Statistical analyses were performed using SPSS version 11 (SPSS Inc., Chicago, IL, USA).

\section{RESULTS}

\section{Patient and tumor characteristics}

Ten cases of gastrointestinal SMT with EUS-FNA results

Table 1. Patients' Baseline Clinical Characteristics

\begin{tabular}{|c|c|c|c|}
\hline & Period $1(n=10)$ & Period $2(n=22)$ & $p$-value \\
\hline Mean age (range, yr) & $63.8(28-85)$ & $68.2(40-84)$ & 0.4 \\
\hline Sex (male/female) & $3 / 7$ & $10 / 12$ & 0.3 \\
\hline Primary tumor site (stomach/duodenum/rectum) & $9 / 1 / 0$ & $16 / 5 / 1$ & 0.4 \\
\hline Mean tumor size (range, $\mathrm{mm}$ ) & $25(13-100)$ & $21(15-60)$ & 0.5 \\
\hline \multicolumn{4}{|l|}{ Final diagnosis } \\
\hline GIST & 10 & 18 & \\
\hline Mucinous adenocarcinoma & 0 & 1 & \\
\hline Neuroendocrine tumor & 0 & 1 & \\
\hline Schwannoma & 0 & 1 & \\
\hline Ectopic pancreas & 0 & 1 & \\
\hline
\end{tabular}

GIST, gastrointestinal stromal tumor. 
Table 2. Technical Results of EUS-FNA for Submucosal Tumors

\begin{tabular}{lccc}
\hline & Period 1 $(\boldsymbol{n}=\mathbf{1 0})$ & Period 2 $(\boldsymbol{n}=\mathbf{2 2})$ & $\boldsymbol{p}$-value \\
\hline Needle size $(19-\mathrm{G} / 22-\mathrm{G})$ at puncture responsible for histologic results & $7 / 3$ & $2 / 20$ & 0.001 \\
Number of needle passes (mean $\pm \mathrm{SD})$ & $5.9 \pm 3.8$ & $3.3 \pm 1.3$ & 0.06 \\
Mean time for procedure (minutes, range) & $24(7-40)$ & $28(12-50)$ & 0.42 \\
Adequate sample for pathological evaluation & $8 / 10(80 \%)$ & $22 / 22(100 \%)$ & 0.03 \\
Diagnostic accuracy & $8 / 10(80 \%)$ & $22 / 22(100 \%)$ & 0.03 \\
Complications & 1 (hematoma) & 0 & 0.3 \\
\hline
\end{tabular}

EUS-FNA, endoscopic ultrasonography-guided fine-needle aspiration.

and subsequent surgical procedures in period 1 and 22 cases in period 2 were identified. Data on the clinical characteristics of the patients and tumors are summarized in Table 1. There were no significant differences in patients' age, sex, tumor location, or tumor size between the two groups. All 10 SMT cases identified in period 1 were diagnosed as GISTs, whereas some tumors identified in period 2 showed non-GIST histological features. One tumor that was diagnosed as mucinous adenocarcinoma had a protruded lesion completely covered with normal mucosa. Two patients with a benign tumor, schwannoma and ectopic pancreas, underwent surgery despite a preoperative diagnosis of a benign tumor by EUS-FNA, because these patients requested surgery due to concerns about malignancy.

\section{Outcomes of EUS-FNA}

The outcomes of EUS-FNA are summarized in Table 2. Specimens sufficient for histological diagnosis could be obtained with a thinner needle (22-G) in all except the initial two cases in period 2, in contrast to the frequent use of a thicker needle in period 1 (use of $19-\mathrm{G}$ needle, $70 \%$ during period 1 vs. $9.1 \%$ during period $2, p=0.001$ ). There was no significant difference between in the procedure duration the two groups ( $p=0.42$ ). Two tumors in period 1 were not diagnosed accurately; one contained benign aspiration cells, whereas the other had insufficient specimens. The overall diagnostic accuracy was significantly higher in period 2 than in period 1 $(100 \%$ vs. $80 \%, p=0.03)$. The number of needle passes by FNA with ROSE by endosonographers tended to be fewer compared to that with FNA without ROSE despite the increased diagnostic accuracy ( $3.3 \pm 1.3$ vs. $5.9 \pm 3.8, p=0.06)$. The only procedure-related complication was hematoma in one patient in period 1 .

\section{DISCUSSION}

The results of the present study demonstrated that ROSE by endosonographers improved the diagnostic yield of gas- trointestinal SMT, particularly for diagnosing GIST. In addition, the procedures were likely to require thinner needles and a reduced number of punctures. ROSE by endosonographers could potentially be a simple solution for obtaining accurate preoperative histopathological diagnosis of GIST.

Currently, it is considered that all GISTs are potentially malignant. When SMT is diagnosed as GIST, the European Society for Medical Oncology (ESMO) and the Japanese GIST guidelines recommend surgical resection. ${ }^{5-8} \mathrm{~A}$ previous report indicated that the sensitivity and specificity of computed tomography (CT) for malignant SMT diagnosis was $80.6 \%$ and $84.2 \%$, respectively. Endoscopy on its own has a suboptimal accuracy rate of $40 \%$ as it identifies malignancy based on submucosal bulges. ${ }^{10}$ Moreover, it is difficult to differentiate GISTs from other SMTs by EUS if there are no high-risk features such as heterogeneity, irregular border, and rapid growth. ${ }^{11}$ Thus, imaging modalities per se were insufficient to make a definitive GIST diagnosis. Therefore, a preoperative pathological diagnosis on all SMT cases is always necessary because some cases may not be GISTs. Because SMTs are covered with mucosa, biopsy findings using conventional biopsy forceps are frequently negative. In this context, EUSFNA is necessary for diagnosis.

It is possible that EUS-FNA has a higher diagnostic performance than EUS based on histopathology in gastrointestinal tract lesions. In 1992, EUS-FNA was first reported to be performed on a pancreatic tumor by Vilmann et al., who used a curved linear array echoendoscope to perform EUS-FNA. ${ }^{12}$ Recently, EUS-FNA has been developed to diagnose SMT, which has led to more accurate preoperative diagnosis of GIST. However, the reported rate of collecting adequate SMT specimens for diagnosis by EUS-FNA was not satisfactory.

Because the diagnosis of GIST by cytology only is likely to be inaccurate, samples with preserved tissue architecture are necessary to make a definitive diagnosis using immunohistochemical staining. Immunohistochemistry tests have revealed that GISTs are positive for c-kit (CD117; 95\%), CD34 (70\%), and SMA (30\%-40\%) and negative for desmin $(<5 \%)$ and S-100 protein $(<5 \%) .{ }^{1}$ In particular, c-kit positivity is 
considered diagnostic for GIST in the setting of a spindle-cell neoplasm.

The reported diagnostic rate of EUS-FNA for GIST ranged from $52 \%$ to $91 \%{ }^{6,13,14}$ In cases with successful immunostaining of obtained specimens in particular, the reported accuracy of preoperative diagnosis of EUS-FNA for surgically resected GIST ranged from $91 \%$ to $100 \%{ }^{14,15}$ This suggests that immunohistochemical analysis with samples obtained by EUS-FNA could be diagnostic and that the potential limitation of this procedure is the difficulty in obtaining sufficient material for immunostaining.

ROSE is a service to determine whether the cells obtained by EUS-FNA are sufficient for pathologists and cytotechnologists to evaluate on site. This study demonstrated significantly improved overall diagnostic accuracy for GIST with ROSE (100\% vs. $80 \%, p=0.03)$. In previous reports, onsite specimen assessment was shown to augment the yield of EUS-FNA in the pancreatobiliary regions. It has been demonstrated that sensitivity (96.2\% vs. 78.2\%) and overall accuracy (96.8\% vs. $86.2 \%)$ of EUS-FNA for pancreatic solid masses is improved with ROSE based on a large retrospective analysis. ${ }^{16}$ The increase in the diagnostic ability by ROSE for SMT in this study could be attributed to the certain procurement of sufficient material for immunostaining, because grossly whitish specimens obtained by EUS-FNA do not always contain sufficient specimens. Moreover, GIST is a hypervascular mass, and it is sometimes difficult to distinguish whitish specimens from coagula and blood. In this regard, ROSE is considered to be more necessary for SMT than for pancreatic tumors.

Despite these advantages of ROSE, it is difficult to ensure that on-site cytopathologists and pathologists are always available in most institutions. In this context, ROSE could be performed by endosonographers in any institution to improve the diagnostic yield of EUS-FNA. In fact, there are two reports showing the usefulness of ROSE by endosonographers with respect to the accuracy of EUS-FNA for pancreatic solid masses. One report concluded that the diagnostic accuracy of ROSE performed by endosonographers and cytopathologists is equivalent. ${ }^{3}$ Another reported that diagnostic accuracy improved from $69.2 \%$ to $91.8 \%$ with the addition of ROSE by endosonographers. ${ }^{4}$ Not needing extra manpower is also an advantage, particularly with regard to the cost. However, it is necessary for endosonographers to undergo training on how to review cytological smears, and the final cytopathological diagnosis has to be referred to cytopathologists.

In this study, the number of needle passes for FNA with ROSE performed by endosonographers tended to be fewer compared with those for FNA without ROSE with increased diagnostic accuracy. This could be attributed to the reduction of unnecessary punctures owing to definitive confirmation of tissue sample procurement by ROSE. In addition, if ROSE indicated that there were inadequate specimens in a puncture location, we could then change the puncture location. Despite the increase in workload in the endoscopy suite, ROSE with endosonographers did not increase the time required for the procedure, indicating that secure procurement of samples could save time wasted on invalid procedures, including ineffective punctures.

Regarding needle size, a large-caliber needle can theoretically acquire a larger amount of cytological and histological specimens, which enables the assessment of architectural features and helps determine malignant potential by immunohistochemical staining. Therefore, EUS-FNA was performed with a 19-G needle for many cases in period 1 . However, thicker needles pose technical difficulties with respect to adequate positioning of the scope and manipulating the needles in angulated positions. In addition, complications are reportedly more frequently encountered with 19-G needles in pancreatic diseases. ${ }^{1718}$ Because ROSE by endosonographers during EUS-FNA with a 22-G needle has been reported to improve diagnostic accuracy for malignant pancreatic tumors, ${ }^{3,4}$ the thinner needle was used for FNA of SMT in period 2, and the diagnostic accuracy obtained was no less than that in the former period. The present results clearly demonstrated that FNA with ROSE for SMT could be performed with smaller-gauge needles.

There are several limitations in this study. The present investigation consisted of a small number of clinical cases with a retrospective single-center design, which introduced a certain bias. Endosonographers who performed ROSE were not blinded to clinical information, and therefore, diagnostic yields may be overestimated. Moreover, as historical controls were used for comparison, the effects of a learning curve for evaluation may not have been completely removed and endosonographers are likely to have improved technically over time. Before generalizing our results, tissue sampling of SMT by EUS-guided fine-needle biopsy (EUS-FNB) should be performed because EUS-FNB is considered the safest and most effective diagnostic modality in tissue sampling of SMT.

In conclusion, this study demonstrated that ROSE by endosonographers could be helpful for preoperative diagnosis of GIST. Hence, we would recommend the use of ROSE by endosonographers during EUS-FNA for diagnosis of GIST, even when no cytopathologists are available.

\section{Conflicts of Interest}

The authors have no financial conflict of interest. 


\section{REFERENCES}

1. Fletcher CD, Berman JJ, Corless C, et al. Diagnosis of gastrointestinal stromal tumors: a consensus approach. Hum Pathol 2002;33:459-465.

2. Erickson RA, Sayage-Rabie L, Beissner RS. Factors predicting the number of EUS-guided fine-needle passes for diagnosis of pancreatic malignancies. Gastrointest Endosc 2000;51:184-190.

3. Hikichi T, Irisawa A, Bhutani MS, et al. Endoscopic ultrasound-guided fine-needle aspiration of solid pancreatic masses with rapid on-site cytological evaluation by endosonographers without attendance of cytopathologists. J Gastroenterol 2009;44:322-328.

4. Hayashi T, Ishiwatari H, Yoshida M et al. Rapid on-site evaluation by endosonographer during endoscopic ultrasound-guided fine needle aspiration for pancreatic solid masses. J Gastroenterol Hepatol 2013;28:656-663.

5. ESMO/European sarcoma network working group. Gastrointestinal stromal tumors: ESMO clinical practice guidelines for diagnosis, treatment and follow-up. Ann Oncol 2012;23 Suppl 7: vii49-vii55.

6. Akahoshi K, Sumida Y, Matsui N, et al. Preoperative diagnosis of gastrointestinal stromal tumor by endoscopic ultrasound-guided fine needle aspiration. World J Gastroenterol 2007;13:2077-2082.

7. Nishida T, Kawai N, Yamaguchi S, Nishida Y. Submucosal tumors: comprehensive guide for the diagnosis and therapy of gastrointestinal submucosal tumors. Dig Endosc 2013;25:479-489.

8. Nishida T, Hirota S, Yanagisawa A, et al. Clinical practice guidelines for gastrointestinal stromal tumor (GIST) in Japan: English version. Int J Clin Oncol 2008;13:416-430.

9. Goto O, Kambe H, Niimi K, et al. Discrepancy in diagnosis of gastric submucosal tumor among esophagogastroduodenoscopy, CT, and endoscopic ultrasonography: a retrospective analysis of 93 consecutive cases. Abdom Imaging 2012;37:1074-1078.

10. Rösch T, Kapfer B, Will U, et al. Accuracy of endoscopic ultrasonography in upper gastrointestinal submucosal lesions: a prospective multicenter study. Scand J Gastroenterol 2002;37:856-862.

11. Shah P, Gao F, Edmundowicz SA, Azar RR, Early DS. Predicting malignant potential of gastrointestinal stromal tumors using endoscopic ultrasound. Dig Dis Sci 2009;54:1265-1269.

12. Vilmann P, Jacobsen GK, Henriksen FW, Hancke S. Endoscopic ultrasonography with guided fine needle aspiration biopsy in pancreatic disease. Gastrointest Endosc 1992;38:172-173.

13. Fernández-Esparrach $\mathrm{G}$, Sendino $\mathrm{O}$, Solé $\mathrm{M}$, et al. Endoscopic ultrasound-guided fine-needle aspiration and trucut biopsy in the diagnosis of gastric stromal tumors: a randomized crossover study. Endoscopy 2010;42:292-299.

14. Ando N, Goto H, Niwa Y, et al. The diagnosis of GI stromal tumors with EUS-guided fine needle aspiration with immunohistochemical analysis. Gastrointest Endosc 2002;55:37-43.

15. Okubo K, Yamao K, Nakamura T, et al. Endoscopic ultrasound-guided fine-needle aspiration biopsy for the diagnosis of gastrointestinal stromal tumors in the stomach. J Gastroenterol 2004;39:747-753.

16. Iglesias-Garcia J, Dominguez-Munoz JE, Abdulkader I, et al. Influence of on-site cytopathology evaluation on the diagnostic accuracy of endoscopic ultrasound-guided fine needle aspiration (EUS-FNA) of solid pancreatic masses. Am J Gastroenterol 2011;106:1705-1710.

17. Karadsheh Z, Al-Haddad M. Endoscopic ultrasound guided fine needle tissue acquisition: where we stand in 2013? World J Gastroenterol 2014;20:2176-2185.

18. Fazel A, Draganov P. Interventional endoscopic ultrasound in pancreatic disease. Curr Gastroenterol Rep 2004;6:104-110 